\title{
Myasthenia Gravis-Our Experience in the Last Three Years with Interesting Associations
}

Sadanandavalli Retnaswami Chandra*, Akshata Huddar, Nibu Varghese, Neeraja Koti, Ganaraja VH, Pooja M and Thomas Gregor Issac

Neurocentre, National Institute of Mental Health and Neurosciences, Bengaluru, India

*Corresponding author: Sadanandavalli Retnaswami Chandra, Neurocentre, National Institute of Mental Health and Neurosciences, Bengaluru, India, Tel: 9449106799; E-mail: drchandrasasi@yahoo.com

Received date: June 09, 2018; Accepted date: July 26, 2018; Published date: July 31, 2018

Copyright: ( 2018 Chandra SR, et al. This is an open-access article distributed under the terms of the Creative Commons Attribution License, which permits unrestricted use, distribution, and reproduction in any medium, provided the original author and source are credited.

\begin{abstract}
Background: Myasthenia gravis is a common immune mediated disease with a prevalence of 1 in 30000 populations. It is eminently treatable but when it presents atypically diagnosis can be missed.

Aim: To look for the common and uncommon clinical features and antibody profile in patients with features of myasthenia gravis.

Settings and design: Myasthenia gravis though well known to be associated with antibody to AchR receptor in the post synaptic membrane, a large group of patients are seronegative for AchR with atypical presentations. AntiTitin antibody titre was done in 7 cases and anti-MUSK in 1 case when strongly indicated by clinical features.

Materials and methods: 29 patients who were confirmed as Myasthenia gravis, seen by authors in the last 3 years were evaluated with anti AchR antibodies in all cases and; Anti-titin and Anti-musk antibodies when clinical indication was strong and patient could afford.

Statistical analysis: Descriptive analysis was done as the number is small.

Results and consideration: Maximum numbers of patients were seen in the younger age group. But patients with more serious disease, patients with Thymic and extrathymic Tumors, Anti-titin and musk associated patients were in the later age group. Thymic tumour has been not detected for nearly 10 years after disease onset in one case. Macroglossia, Motor neuron disease overlap is seen in one case which is relatively very rare. Though Myasthenia gravis is seen commonly in the young persons, Thymic and extrathymic tumors, Anti-titin antibody associated Myasthenia gravis is seen in later age group. Macroglossia and Motor neuron disease overlap features do not exclude MG.
\end{abstract}

Keywords: Myasthenia gravis; Anti-titin antibodies; Macroglossia; Motor neuron disease; pancreatic tumor

\section{Key message:}

Macroglossia and Motor neuron disease overlap features do not exclude MG. when anti-titin antibody is positive other neoplasms including pancreatic should be looked for.

\section{Introduction}

Myasthenia gravis (MG) is an autoimmune disease with genetic association with polymorphism concerned with autoimmunity and has a very variable clinical spectrum, course and response to treatment [1]. Generally it is a female dominant disease affecting all age groups with minor variations in etio-pathogenesis and course. The common antibodies seen are against nicotinic acetylcholine receptors and other associated proteins, Muscle specific tyrosine kinase (musk), low density lipoprotein receptor, anti-striated muscle, Actin, a-actinin, Rynodin, Myosin, Connectin and Titin [2]. Anti-musk antibody associated myasthenia presents as severe type of oculobulbar disease affecting females in middle age however long term outcome is comparable to that of anti AchR antibody associated type.

Titin is a giant filamentous protein which is spread all over sarcomere and is responsible for elasticity. Antibody to this protein is restricted to a small region MGT30 which is otherwise called myasthenia gravis with thymoma $30 \mathrm{kd}$ [3]. Autoimmune diseases associated with myasthenia are Rheumatoid arthritis, Thyroid diseases, SLE with reported incidence of 8.7\%-25\% [4]. Diagnosis is done based on clinical features of fluctuating ocular, bulbar and or limb weakness, electrophysiological study using repetitive nerve stimulation, SFEMG when possible and serological tests [5]. Association with generalized autonomic dysfunction, tumors are also reported [6]. Severity is often graded using modified Osserman grading to five grades varying from 1 to 5. There is a recent method Myasthenia Gravis Impairment Index (MGII) which is more practical is also available [7,8]. Treatment consists of symptomatic measures as well as disease modifying treatment using steroids followed by steroid sparing agents, plasma exchange, and intravenous immunoglobulin. Whenever underlying thymoma or other neoplasm is identified surgery is indicated. Patients should avoid drugs which cause neuromuscular transmission impairment, special precaution is needed during pregnancy to mother 
Citation: Chandra SR, Huddar A, Varghese N, Koti N, Ganaraja VH, et al. (2018) Myasthenia Gravis-Our Experience in the Last Three Years with Interesting Associations. J Gen Pract (Los Angel) 6: 363. doi:10.4172/2329-9146.1000363

Page 2 of 4

and new-born baby, attention to choice of anesthetic agents during surgery, avoiding muscle relaxants are important [9-11].

Myasthenia can overlap with anterior horn cell disease though rare and has to be suspected when patients show resistance to treatment with associated wasting and fasciculation's supported by electrophysiological tests. Only 25 such cases are reported in literature and it paves way to a possibility that immunopathology and neuromuscular junction impairment may be there in amyotropic lateral sclerosis too [12]. Tongue muscles are generally not affected in myasthenia but rarely atrophic tongue, trident tongue and lateral tongue force reduction are reported $[13,14]$. There is a single case report of swollen tongue in myasthenia gravis in literature [15]. Extrathymic tumors and second malignancy in patients with thymic tumors are seen with older age they can occur 20 years before to 35 years after the diagnosis of myasthenia and older age and immune dysregulation are considered as causes [16,17].

\section{Patients and Methods}

29 patients seen by the authors in the last 3 years who satisfied criteria for diagnosis of myasthenia gravis were evaluated with detailed clinical assessment, haematological evaluation for routine as well as thyroid function tests, rheumatoid factor, AchR antibody, CT mediatinum, USG abdomen, electrophysiological testing with repetitive nerve stimulation sampling Nasalis, Trapezius and Deltoid in all patients. Paraneoplastic work up was done in seven patients based on the clinical suspicion due to severe weight loss, very late onset and poor treatment response. Anti-musk antibody was done in a single case who presented with repeated crisis. CT abdomen was done in selected cases.

\section{Results}

29 patients were seen by authors in last 3 years. There were 10 females and 19 males. The age group is as follows. In the 20 to 40 years group there were 15 patients. In the 41 to 60 years group there were 10 patients. In the 60 and above group there were 4 patients (Figure 1).

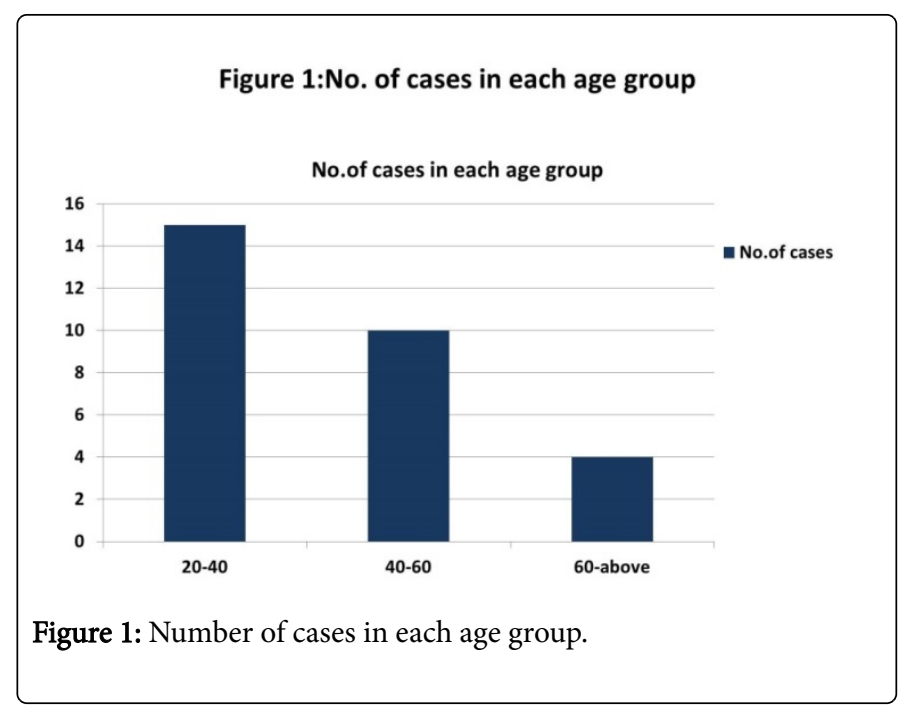

J Gen Pract (Los Angel), an open access journal ISSN:2329-9126

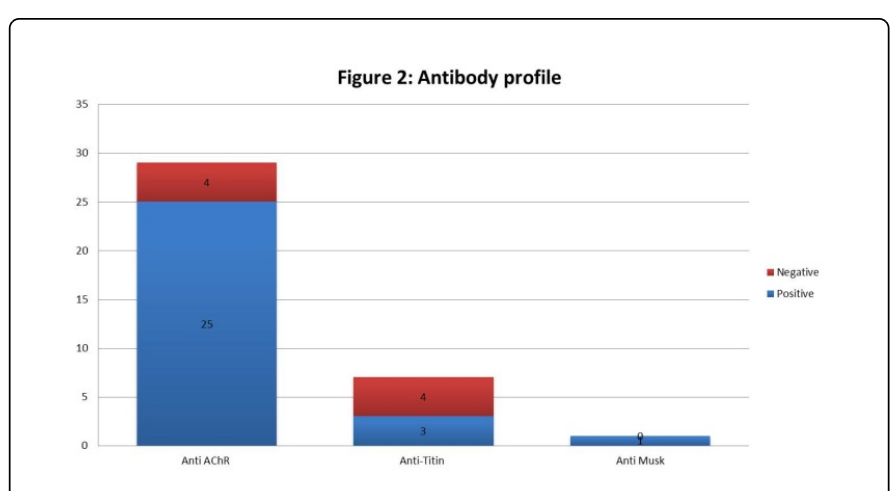

Figure 2: Antibody profile.

Anti AchR antibodies were positive in 25 patients. Anti-musk was done in one patient and it was positive. Anti-titin antibody was done in 7 patients and it was strongly positive in 4 patients (Figure 2).

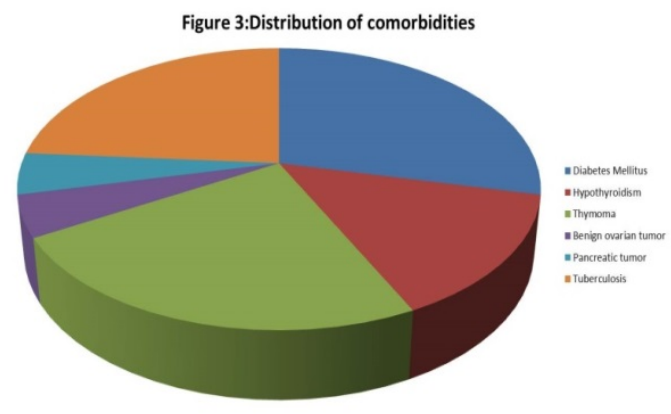

Figure 3: Distribution of comorbidities.

The other co morbidities seen were diabetes mellitus in 6 patients, pulmonary tuberculosis in 5 patients and hypothyroidism in 3 patients (Figure 3).

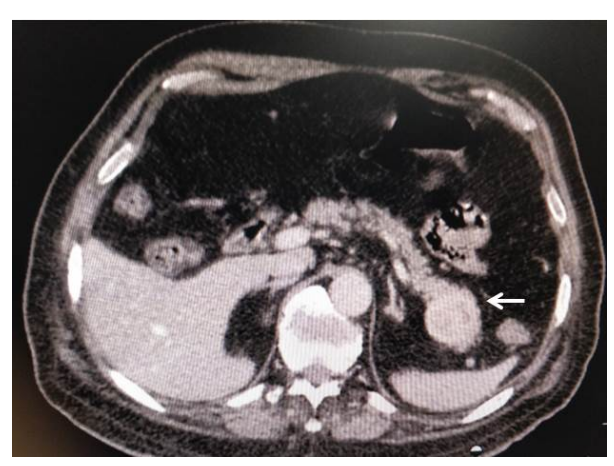

Figure 4: $\mathrm{CT}$ abdomen showing pancreatic tail tumour.

Thymoma was seen in 5 patients and only one was reported malignant. Benign ovarian tumor was seen in one patient and pancreatic tumor was seen in one patient (Figure 4).

All patients with thymic mass underwent surgery. Patient with malignant thymoma underwent radiotherapy. Patient with ovarian 
mass chose not to take the risk of anesthesia. Patient with pancreatic tumor was in 7 th decade and decision had not been made so far regarding surgery.

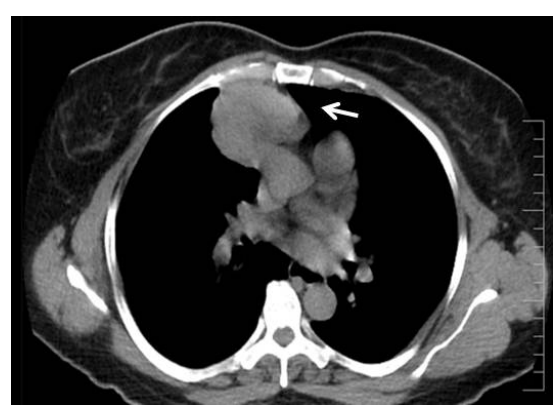

Figure 5: CT thorax showing thymic mass.

The mean duration of illness at which the patients presented to our institute was about 4.5 months. All patients were confirmed as myasthenia gravis with clinical, electrophysiological and radiological methods as per the criteria laid down by the task force for diagnosis of myasthenia [18]. The severity of the patients at presentation was grade 5 in $3 \%$, grade 4 in $3 \%$ and grade $2-3$ in $94 \%$ as per Jaretzki et al. [19]. Maximum severity was seen within 1 year in $94 \%$ of the patients and at 10 years in $3 \%$ and as presenting symptom in $3 \%$. Out of 4 patients with strongly positive Anti-titin antibody, 3 patients had benign Thymoma (Figure 5).

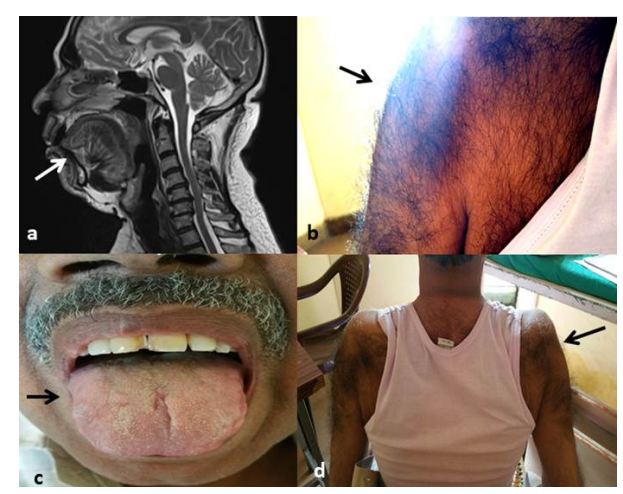

Figure 6: As indicated by arrows (a) Macroglossia in Saggital MRI image (b) Hypertrichosis (c) Clinical picture of the big tongue with healed scars following biting (d) Muscle wasting.

Among them one patient who is a 62 years old gentleman had unique features. He was diagnosed as suffering from myasthenia gravis and was in fairly good remission with steroids, Rituximab, Azathioprine for more than 10 years. For the last five years he experienced repeated biting of tongue and developed repeated tongue injury to an extent that on one occasion he needed suturing of tongue. One year ago patient noticed inability to lift left upper limb which was slowly progressing. At the time of arrival to our center patient was having slurred speech, tongue was bulky on the right side and thin on the left side, which showed injury marks, hypertrichosis of back, severe wasting of supraspinatus, infraspinatus, deltoid, biceps, triceps, left more than right (Figure 6).
There was also thinning of muscles of left leg compared to right. There were fasciculation's in the deltoid. Reflexes were depressed in upper limbs and just elicitable in lower limbs and these features were suggestive of anterior horn cell involvement. Patients were treated as follows: Thymectomy was done in all patients with thymic tumour. Patients who presented in Grade 4-5 were initiated with plasmapharesis, assisted ventilation and symptomatic measures with parenteral neostigmine and immunosuppression. The patients in grade 2-3 received pyridostigmine tablets to a maximum of 5 tablets per day based on the response. All of them received Azathioprine $1 \mathrm{mg} / \mathrm{kg}$ along with oral prednisolone started with $5 \mathrm{mg}$ and slowly increased till full remission was achieved. Later steroids were tapered off over 3 months and put on $7.5 \mathrm{mg}$ as maintenance.

One patient who had anti-MUSK antibody positive presented with repeated crisis in the first two years and then currently stable for one year. We did not have any mortality in this group during the follow up period.

\section{Discussion}

This group of patients with myasthenia gravis revealed the following. Anti-titin antibodies were done in patients who had short duration of illness with late onset and thymic tumors. Anti- musk was done in the patients who presented with grade 5 disease. The limiting factor in other patients was the financial constraints. Patient with antimusk antibodies presented with repeated crisis that is stage 5 disease [16]. $80 \%$ of Anti-titin associated patients present with thymoma. Thymoma might become evident as late as 10 years after the disease onset. One of our Anti-titin antibody positive patients showed unique feature like macroglossia, hypertrichosis and muscle wasting. Whether there is a role for anti-titin antibody in these overlap cases needs to be evaluated. Only a single case of macroglossia in myasthenia is reported in literature. The common conditions associated with macroglossia are amyloidosis, hypothyroidism, mucopolysaccaridoses, glycogen storage disease and neurofibromatosis, infant of diabetic mother, vascular malformations of the tongue, lymhangioma, haemangioma and unique muscular dystrophy like Beckwith-Wiederman syndrome [20].

\section{Consideration}

This small group of patients with myasthenia gravis revealed unique features like its association with anterior horn cell involvement, macroglossia and hypertrichosis in anti-titin antibody positive cases. This has to be kept in mind when patients stop responding even years after stable phase and they need to be re-evaluated. Anti-musk associated patients present with more serious disease and course to start with but stabilize over time. Benign tumors are more common both in thymus and in other areas.

\section{Limitation}

This group involves a limited number of patients seen by the authors and may not be representative of the whole population. General prevalence of systemic malignancy has not been compared.

\section{References}

1. Wang N, Shen N, Vyse TJ, Anand V, Gunnarson I, et al. (2011) Selective IgA deficiency in autoimmune diseases. Mol Med 17: 1383-1396. 
Citation: Chandra SR, Huddar A, Varghese N, Koti N, Ganaraja VH, et al. (2018) Myasthenia Gravis-Our Experience in the Last Three Years with Interesting Associations. J Gen Pract (Los Angel) 6: 363. doi:10.4172/2329-9146.1000363

Page 4 of 4

2. Liu FC, Kuo CF, See LC, Tsai HI, Yu HP (2017) Familial aggregation of myasthenia gravis in affected families: A population-based study. Clin Epidemiol 9: 527-535.

3. Aarli JA (2001) Titin, thymoma, and myasthenia gravis. Arch Neurol 58: 869-870.

4. Fang F, Sveinsson O, Thormar G, Granqvist M, Askling J, et al. (2015) The autoimmune spectrum of myasthenia gravis: A swedish population-based study. J Intern Med 277: 594-604.

5. Mahadeva B, Phillips LH, Juel VC (2008) Autoimmune disorders of neuromuscular transmission. Semin Neurol 28: 212-227.

6. Puneeth CS, Chandra SR, Yadav R, Sathyaprabha TN, Chandran S (2013) Heart rate and blood pressure variability in patients with myasthenia gravis. Ann Indian Acad Neurol 16: 329-332.

7. Barnett C, Bril V, Kapral M, Kulkarni A, Davis AM (2016) Development and validation of the myasthenia gravis impairment index. Neurol 87: 879-886.

8. de Perrot M, Liu J, Bril V, McRae K, Bezjak A, et al. (2002) Prognostic significance of thymomas in patients with myasthenia gravis. Ann Thoracic Surg 74: 1658-1662.

9. Lacomblez L, Warot D (1990) Myasthenia gravis and drugs. Therapie 45: 273-275.

10. Mitchell PJ, Bebbington MI (1992) Myasthenia gravis in pregnancy. Obstetr Gynecol 80: 178-181.

11. Baraka A (1992) Anaesthesia and myasthenia gravis. Can Journal Anaesth 39: 476-486.

12. Johnson IP, Longone P (2017) Commentary: Amyotrophic lateral sclerosis and myasthenia gravis overlap syndrome: A review of two cases and the associated literature. Frontier Neurol 8: 356.
13. Burch J, Warren-Gash C, Ingham V, Patel M, Bennett D, et al. (2006) Myasthenia gravis-a rare presentation with tongue atrophy and fasciculation. Age and ageing 35: 87-88.

14. Weijnen FG, Kuks JB, Van Der Bilt A, Van Der Glas HW, Wassenberg MW, et al. (2000) Tongue force in patients with myasthenia gravis. Acta Neurol Scand 102: 303-308.

15. Davison SP, McDonald TJ, Wolfe ME (1997) Swollen tongue: A presentation of myasthenia gravis. Otolaryngol Head Neck Surg 116: 244-246.

16. Guptill JT, Sanders DB, Evoli A (2011) Anti-musk antibody myasthenia gravis: Clinical findings and response to treatment in two large cohorts. Muscle Nerve 44: 36-40.

17. Monden Y, Uyama T, Kimura S, Taniki T (1991) Extrathymic malignancy in patients with myasthenia gravis. Eur J Cancer 27: 745-747.

18. Yoshikawa H, Iwasa K, Kawaguchi N, Kira J, Kohriyama T, et al. (2017) Assured diagnostic critera of myasthenia gravis by the task force for evidence-based diagnostic and therapeutic algorithms for the national policy of intractable diseases of Japan. J Neurol Sci 381: 1086.

19. Jaretzki A, Barohn RJ, Ernstoff RM, Kaminski HJ, Keesey JC, et al. (2000) Myasthenia gravis: Recommendations for clinical research standards. Neurol 55: 16-23.

20. Filippi G, Mckusick VA (1970) The Beckwith-Wiedemann syndrome: The exomphalos-macroglossia-gigantism syndrome report of two cases and review of the literature. Medicine 49: 279-298. 